\title{
Evaluación de la viabilidad del Lactobacillus spp. encapsulado en maltodextrina, como probiótico en un jugo de Lepidium meyenni walp
}

\author{
Evaluation of the viability of Lactobacillus spp. encapsulated in \\ maltodextrin, as a probiotic in a Lepidium meyenni walp juice
}

\author{
a Julca Marcelo, E.; Huaraca Meza, F; \\ Rojas Ticse, A.; Lazarte Pariona, F; Oré Mallma, R. \\ Facultad de Ingeniería y Ciencias Humanas / Universidad Nacional del Centro del Perú \\ Email: ejulca@uncp.edu.pe
}

\section{Resumen}

El trabajo de investigación evaluó; en primer lugar, la temperatura del aire $\left(120,140{ }^{\circ} \mathrm{C}\right)$ durante el proceso de microencapsulación por el método de atomización; se evaluaron, también, tres concentraciones de maltodextrina $(15,25$ y $30 \%)$ empleado como agente encapsulante del Lactobacillus spp. en el jugo reconstituido de harina de maca, obteniendo como resultados que a una temperatura de $120{ }^{\circ} \mathrm{C}$ y una concentración de $15 \%$ de maltodextrina, la eficiencia de encapsulado fue de 83,57\% de Lactobacillus spp. que al ser comparado con el Lactobacillus spp. libre reporta una gran diferencia en el porcentaje de sobrevivencia que fue del 57,70 \%. Además, se determinó que después de 3 semanas de almacenamiento a $4{ }^{\circ} \mathrm{C}$, el Lactobacillus spp. encapsulado a una temperatura de 120 ${ }^{\circ} \mathrm{C}$ y una concentración de $15 \%$ de maltodextrina, tuvo una concentración final de bacterias de $8,18 \mathrm{Log}$ ufc/ml llegando a un porcentaje de sobrevivencia de 82,23\%.

Posteriormente, se realizó la comparación con el Lactobacillus spp. no encapsulado a la misma temperatura de almacenamiento, obteniéndose una concentración final de bacterias de 4,24 Log ufc/ml que representa un porcentaje de sobrevivencia de 47,64\%. Al realizar la simulación de las condiciones del tracto gastrointestinal para ver la sobrevivencia en el jugo gástrico a diferentes $\mathrm{pH}$, se observa que el mayor porcentaje de sobrevivencia se da cuando el pH del JGS (jugo gástrico simulado) es 2 y el pH del JIS (jugo intestinal simulado) es 7; obteniéndose un recuento de 7,2041 Log ufc/ml de sobrevivencia.

Palabras clave: atomización y encapsulación, probiótico, potencial agroindustrial, seguridad alimentaria

\begin{abstract}
The first work evaluated on the investigation of air temperature $\left(120,140{ }^{\circ} \mathrm{C}\right)$ during the process of microencapsulation for the method of atomization, three components of maltodextrin (15, 25 and $30 \%$ whish is used as an encapsulation agent for Lactobacillus spp, were also evaluated in the juice reconstituted of flour of bruise; obtaining as results that to a temperature of $120{ }^{\circ} \mathrm{C}$ and a concentration of $15 \%$ of maltodextrina, the efficiency of encased was $83,57 \%$ of Lactobacillus spp. that on having been compared with the free Lactobacillus spp. brings(reports) a great difference in the percentage of survival that was $57,70 \%$. In addition, it was determined that after 3 weeks of storage to $4{ }^{\circ} \mathrm{C}$, the Lactobacillus spp. encased to a temperature of $120^{\circ} \mathrm{C}$ and a concentration of $15 \%$ of maltodextrina, had a final concentration of bacteria of $8,18 \mathrm{Log}$ ufc/ $\mathrm{mL}$ coming to a percentage of survival of $82,23 \%$.

Later, comparison was realized with the non encased Lactobacillus spp. at the same storage temperature, resulting in a final concentration of bacteria of 4,24 Log ufc/ $\mathrm{ml}$ representing a survival rate of $47,64 \%$. when making realized the simulation of the conditions of the gastrointestinal tract to see the survival in the gastric juice to different $\mathrm{pH}$, it is observed that the major percentage of survival is given when the $\mathrm{pH}$ of the JGS (gastric simulated juice) is 2 and the $\mathrm{pH}$ of the JIS (intestinal simulated juice) is 7; with a 7,2041 Log ufc/ml survival count.
\end{abstract}

Keywords: atomization and encapsulation, probiotic, agro-industrial potential, food safety 


\section{Introducción}

Para apreciar el potencial agroindustrial, es necesario analizar diversos aspectos; entre ellos, la existencia de una gran biodiversidad que constituye, sin lugar a dudas, una de las mayores riquezas, sino la mayor, con que cuenta la provincia de Junín.

Los variados ecosistemas se traducen en diversidad ecológica, la alta diversidad de especies, la diversidad de recursos genéticos y humanos, que conforman nuestra biodiversidad, proporcionándole ventajas comparativas a la provincia de Junín.

La utilización racional y sostenible de estos recursos con empresas orientadas al ecoturismo, la industria farmacéutica, la agroindustria, la acuicultura y otros, es importante para lograr ventajas competitivas con productos de mayor valor agregado. Precisamente, en el caso de la agroindustria, herramienta clave para el desarrollo, es que se defina, clasifique y señale su importancia, se da a conocer un sistema integrado de transformación, que reúne a la producción, procesamiento, comercialización, gestión y los aspectos socio-económicos de la cadena productiva. Esto es posible en el marco de los bionegocios, concepto que comprende objetivos generales como son: la conservación de los recursos, la sustentabilidad y la justa y equitativa distribución de las ganancias generadas. Se debe elaborar entonces proyectos rentables, desde un punto de vista económico y ambiental.

Los recursos naturales de la provincia de Junín, se encuentran distribuidos en la flora y fauna. La selección de estas especies a partir del universo conocido, se presentan en un listado por rubros de productos, no siendo necesariamente excluyente para el caso de determinados productos introducidos y, destacando los siguientes: tintes y colorantes, plantas medicinales, especias y hierbas aromáticas, frutas nativas, cereales y granos, raíces y tubérculos, fibras, biopesticidas, y otros.

Luego se ha priorizado las especies en función de criterios, como la exclusividad de productos autóctonos y de la potencialidad agroindustrial a futuro, encontrándose prioridad estratégica en los siguientes: maíz morado, maca, quinua, sauco, barbasco, fibra de vicuña, entre otros.

Dentro del marco general de limitaciones encontradas, son expuestos los puntos críticos (taxonomía, caracterización, control de calidad, investigación farmacológica y clínica, manejo de cultivos y crianzas, post-cosecha, transformación, marketing y desarrollo de productos, información y ecología) de los futuros proyectos, con miras a ser abordados en trabajos sistemáticos de investigación aplicada con visión de mediano y largo plazo. La síntesis de la problemática de cada rubro, da lugar a una discusión de las estrategias concretas de desarrollo agroindustrial, con ejemplos de productos específicos.
La oferta diversa de productos agrícolas de la provincia de Junín al mundo, solo tendrá posibilidades sostenibles, al apoyarse en la agroindustria y el asesoramiento de profesionales competentes, las ventajas comparativas de la biodiversidad, puedan ser aprovechadas eficientemente en el marco de los bionegocios, generando no sólo rentabilidad económica, sino, y quizás esto sea lo más importante, mejorando la calidad de vida de la población actual y simultáneamente conservando los recursos naturales para las generaciones futuras.

\section{Antecedentes}

Los estudios de potencial agroindustrial en nuestro país son muy limitados, en referencia a países como Chile, Colombia, Brasil y México en los que son comunes encontrar grandes volúmenes de información con respecto a ello; es por eso, que se puede ligar este hecho al desarrollo agroindustrial de los países, mientras, para los anteriormente mencionados, la agroindustria constituye el pilar fundamental del desarrollo. También, se puede destacar que la FAO, a través del documento Agroindustrias para el Desarrollo, indica en la parte introductoria que: "El desarrollo de agroindustrias competitivas es crucial para generar oportunidades de empleo e ingresos. Contribuye, además, a mejorar la calidad de los productos agrícolas y su demanda. Las agroindustrias tienen el potencial de generar empleo para la población rural, no sólo a nivel agrícola, sino también, en actividades fuera de la explotación como manipulación, envasado, procesamiento, transporte y comercialización de productos alimentarios y agrícolas. Existen señales claras de que las agroindustrias están teniendo un impacto global significativo en el desarrollo económico y la reducción de la pobreza, tanto en las comunidades urbanas como rurales. Sin embargo, muchos países en desarrollo (especialmente en África) todavía no se han dado cuenta de todas las posibilidades que ofrecen las agroindustrias como motor de desarrollo económico...” (Da Silva et al., 2013).

De otra parte, es importante conocer que la demanda de alimentos y productos agrícolas está sufriendo cambios sin precedentes. El aumento tanto del ingreso per cápita, como de la urbanización y del número de mujeres trabajadoras, ha incrementado la demanda de materias primas de alto valor de productos procesados y de alimentos preparados. Existe una clara tendencia hacia dietas, que incluyen una mayor cantidad de alimentos de origen animal, como pescado, carne y productos lácteos, que aumenta a su vez la demanda de cereales para pienso (FAO, 2007). También, se está dando un uso creciente de productos agrícolas, especialmente granos y cultivos oleaginosos, como materia prima para la producción de bioenergía. El comercio internacional y las comunicaciones, están acelerando los cambios en la demanda, lo que conlleva la convergencia de patrones dietéticos y un creciente interés en comidas étnicas de lugares geográficos específicos. 
La naturaleza y alcance de la estructura cambiante de la demanda agroalimentaria, ofrecen oportunidades sin precedentes para la diversificación y adición de valor en el sector 3 agrícola, especialmente en los países en desarrollo. Como reflejo del cambio de demanda por parte de los consumidores, la década de 1990 fue testigo de una diversificación de la producción en los países en desarrollo hacia frutas y hortalizas no tradicionales. La participación de los países en desarrollo en el comercio internacional de frutas y hortalizas no tradicionales, ha aumentado rápidamente en los últimos años (FAO, 2007).

Según Rabobank (2008), las ventas anuales de alimentos procesados a nivel mundial, se estiman actualmente en más de 3000 MUSD o, lo que es lo mismo, en aproximadamente tres cuartos de las ventas totales de alimentos a nivel internacional. Si bien la mayoría de estas ventas se producen en los países de ingresos altos, en los últimos 25 años, los países en desarrollo han generado un porcentaje de casi el doble de adición de valor en la producción global de las principales categorías de productos fabricados por las agroindustrias (FAO, 2007).

Las perspectivas de un crecimiento constante de la demanda de alimentos y de productos agrícolas con valor añadido constituyen un incentivo para prestar mayor atención al desarrollo de las agroindustrias en un contexto de crecimiento económico, seguridad alimentaria y estrategias para acabar con la pobreza. Es conocido que las agroindustrias, entendidas aquí como un componente del sector manufacturero en el que la adición de valor a las materias primas agrícolas deriva de operaciones de procesamiento y manipulación, son motores eficientes de crecimiento y desarrollo.

Con sus vínculos progresivos y regresivos, las agroindustrias tienen altos efectos multiplicadores en términos de creación de empleo y de adición de valor. Por ejemplo, una nueva planta de procesamiento de leche no solo crea empleos en sus propias instalaciones de transformación, sino también, en las granjas lecheras, los centros de recogida de leche, los suministradores de insumos agrícolas y la distribución. El aumento de la demanda creado por una empresa agroindustrial, estimula los negocios más allá de los vínculos con sus proveedores directos de insumos y sus compradores; es decir, una completa gama de servicios auxiliares y actividades de soporte en los sectores secundario y terciario de la economía también se verán impactados de manera positiva. Es más, dado que generalmente los productos agrícolas se caracterizan por ser perecederos y voluminosos, muchas plantas agroindustriales y pequeñas empresas de agroprocesamiento tienden a ubicarse cerca de las fuentes principales de materias primas; de esta manera, ejercen un impacto socioeconómico inmediato en las áreas rurales.

El Informe sobre el desarrollo mundial 2008 (Banco Mundial, 2007) llamó la atención sobre el hecho que, aproximadamente 800 millones de personas están consideradas como pobres y viven con ingresos de menos de un dólar al día. Un $75 \%$ de los pobres del mundo, viven en áreas rurales y la agricultura es su principal fuente de subsistencia. Luchar contra la pobreza requerirá llevar el desarrollo y el crecimiento económico a las áreas rurales. La agroindustria, es parte de la respuesta a ese desafío.

El crecimiento acelerado de las agroindustrias en los países en desarrollo también presenta riesgos en términos de equidad, sostenibilidad e inclusión. Cuando existe un poder de mercado desequilibrado en las agrocadenas, la adición y la captación de valor pueden estar concentradas en uno o pocos participantes de la cadena, perjudicando a los demás. Las agroindustrias solo serán sostenibles si son competitivas en términos de costes, precios, eficiencia operativa, oferta de productos y otros parámetros asociados, y solo si la remuneración que reciben los agricultores es lucrativa para estos. Establecer y mantener la competitividad constituye un desafío particular para las pequeñas y medianas empresas agroindustriales y para los pequeños agricultores.

A pesar que la agroindustria tiene el potencial de proporcionar una salida fiable y estable para los productos agrícolas, la necesidad de garantizar la competitividad favorece a aquellos agricultores que son capaces de entregar productos en mayor cantidad y de mejor calidad.

Puesto que los agricultores más pequeños y con menos recursos quedan excluidos de las cadenas de abastecimiento, los beneficios socioeconómicos de las agroindustrias quedan potencialmente reducidos. Por ello, es necesario contar con políticas y estrategias que promuevan las agroindustrias y, al mismo tiempo, consideren los temas de competitividad, equidad e inclusión.

El rápido aumento de los precios de los alimentos observado en 2007 y 2008, fue un crudo recordatorio de que la naturaleza cambiante de los sistemas agroalimentarios y la manera en que los responsables de las políticas responden a los cambios, pueden tener consecuencias humanitarias y políticas directas. Los ajustes en el sector agrícola y las agroindustrias en los años 1990 y comienzos del 2000 contribuyeron a reducir las reservas internacionales y la oferta de alimentos de primera necesidad.

El sistema alimentario mundial no fue capaz de responder a un cúmulo de eventos adversos que impactaron sobre la oferta y la demanda a corto plazo. Como consecuencia del repunte de precios de los alimentos y la escasez, las respuestas políticas que incluyeron la prohibición de las exportaciones empeoraron aún más una situación de mercado ya desequilibrada. La crisis de los 4 precios de los alimentos, aunque ya ha remitido, señala la importancia de las recientes tendencias en los sistemas agroalimentarios, así como la necesidad de políticas y estrategias sólidas que mejoren la competitividad y el impacto en el desarrollo de la agroindustria. 


\section{Métodos y materiales}

\section{Población y muestra}

La población estuvo constituida por todos los recursos animales y vegetales de la provincia de Junín. Se estimó, como muestra, sectores de terreno de la provincia en la que se debía de identificar las especies existentes.

\section{Disponibilidad de recursos}

De junio a noviembre, se procedió a identificar las especies con potencial agroindustrial dentro de la provincia de Junín, los mismos que luego fueron analizados en función de las ventas, exportaciones o la demanda de los mercados a nivel nacional y mundial. Fue también necesario realizar un análisis más concienzudo a fin de determinar si era posible su producción a mayor escala dentro de nuestro territorio respetando siempre los recursos suelo, agua y aire, para brindar seguridad alimentaria.

\section{Resultados y discusión}

Los rubros a ser considerados como fuentes potenciales para el desarrollo agroindustrial de la provincia de Junín comprenden los siguientes grupos:

\section{Tintes y colorantes naturales}

- Achiote (Bixa orellana)

- Maíz morado (Zea mays)

\section{Plantas medicinales}

- Ajo sacha (Mansoa alliacea)

- Congona (Peperomia sp.)

- Copaiba (Copaifera officinalis)

- Hercampure (Gentianella tomentosa)

- Huanarpo (Jatropha sp.)

- Maca (Lepidium meyenii)

- Paico (Chenopodium ambrosioides)

Especias y hierbas aromáticas

- Aji (Capsicum annuum)

- Rocoto (Capsicum pubescens)

- Huacatay (Tagetes minuta)

- Molle (Schinus molle)

- Paico (Chenopodium ambrosioides)

- Sacha culantro (Eryngium foetidum)

\section{Estimulantes}

- Boldo (Peumus boldo)

\section{Frutales nativos}

- Naranjilla o Lulo (Solanum quitoense)

- Aguaymanto (Phisalis peruviana)

- Lúcuma (Pouteria lucuma)

- Saúco (Sambucus peruviana)

- Tomate de árbol (Cyphomandra betacea)

- Tumbo serrano (Passiflora mollis)

\section{Cereales y granos andinos}

- Maíz (Zea mays)

- Quinua (Chenopodium quinoa)

\section{Raíces y tubérculos}

- Maca (Lepidium meyenii)

- Mashua (Tropaelum tuberosum)

- Oca (Oxalis tuberosa)

- Olluco (Ullucus tuberosus)

- Papa (Solanum tuberosum)

- Yacón (Smallanthus sonchifolius)

\section{Fibras naturales}

- Alpaca (Lama vicugna)

- Llama (Lama guanicoe)

- Vicuña (Vicugna vicugna)

- Ovino (Ovis orientalis aries)

\section{Biopesticidas y otros}

- Muña (Minthostachys cetosa)

- Tara (Caesalpinia tintorea)

\section{Agricultura orgánica}

- Maíz amiláceo (Zea mays)

- Piña (Ananas comosus)

- Banano

- Café

\section{Animales}

- Alpaca (Lama vicugna)

- Cuy (Cavia tschudii)

- Llama (Lama guanicoe)

- Ovino (Ovis orientalis aries)

- Vacuno (Bos primigenius taurus)

\section{Peces y crustáceos}

- Ranas (Batrachophrynus sp. y Telmatobius culeus)

- Truchas (Oncorhynchus mykiss)

\section{Limitantes}

Las ventajas comparativas que presenta nuestro país no se traducen necesariamente en ventajas competitivas; es decir, no se logra capitalizar las posibilidades que nos ofrece el potencial de la biodiversidad. Dentro de las limitantes concretas que se presentan en la práctica para el desarrollo agroindustrial, se mencionan:

1. Producción de materias primas insuficiente. Muchas veces exportamos basados en la recolección de material silvestre, con las limitaciones inherentes que esto trae. (Ej: caso de la maca)

2. Calidad de las materias primas no homogéneas, sin certificar.

3. Falta de continuidad de las fuentes para el abastecimiento regular. Incumplimiento muchas veces de los envíos de exportación.

4. Insuficiente o nulo tratamiento de la ecología y conservación del medio ambiente.

5. No se ha logrado interiorizar en el gremio agroindustrial la necesidad de compartir con la responsabilidad de invertir en el manejo adecuado del medio ambiente. 
La pregunta que surge entonces es ¿cómo lograr ventajas competitivas en agroindustria a partir de la biodiversidad? La respuesta que se encuentra a este objetivo la se puede sintetizar en el planteamiento y la ejecución de proyectos de inversión de mediano y largo plazo que contemplen el trabajo sistemático de investigación aplicada, dirigidos a superar cada uno de los puntos críticos de la cadena productiva.

\section{Puntos críticos}

a) Taxonomía. El conocimiento de las especies y su caracterización requieren de la plena identificación botánica sistemática. En muchos casos existe el desconocimiento exacto de la especie o variedad con que nos disponemos a trabajar. Resulta necesario, plantear la revisión taxonómica, la identificación y, en el caso de material silvestre, realizar un inventario con prioridad de las especies seleccionadas, como requisito indispensable de todo proyecto de desarrollo.

b) Caracterización. El conocimiento cabal de las características físico-químicas, nutricionales, organolépticas, de sanidad e higiene, forman parte de la necesaria caracterización de los productos frescos, semiprocesados y terminados.

c) Control de calidad y la certificación. Los mercados en general, y particularmente los de países desarrollados, son cada día más exigentes en cuanto a calidad de productos se refiere. Uno de los aspectos indispensables, es contar con las normas técnicas de calidad y con las metodologías de análisis necesarias para cumplir con todos los requisitos demandados para un producto determinado. El respeto de la legislación vigente es necesario en cada caso. La certificación de los productos permite una mejor promoción y comercialización de los productos ofertados. Un ejemplo de certificación, constituye el caso de los denominados productos orgánicos o ecológicos, estos obtienen un plus en el precio de compra, debido la preferencia manifiesta en la demanda de los mercados naturistas.

d) Investigación farmacológica y clínica en el caso de productos medicinales. Los resultados de las bondades preventivas y curativas de los productos deben estar respaldados por estudios científicos, clínicos y farmacológicos. De esta manera, se logra la validación de las aplicaciones específicas y la posibilidad de obtener valor agregado que compense la inversión.

e) Manejo de cultivos y crianzas. La necesidad del aumento de la producción y de la productividad, así como el manejo y uso racional de los recursos, constituyen el mayor fundamento para el manejo técnico de cultivos y crianzas. Para cultivos, estos incluyen actividades de propagación, manejo de podas, abonamiento, riego, marco de plantación, control de plagas, entre otras. Para crianzas, se cita, el mejoramiento genético, el sistema de crianza, la alimentación, etc. f) Manejo de post-cosecha. En la actualidad, el Perú viene desarrollando la agroindustria y agroexportación, sustentado en la comercialización de productos dirigidos hacia el mercado nacional e internacional, cada día más exigentes en términos de calidad, homogeneidad, volúmenes y continuidad del abastecimiento. La necesidad de prolongar el periodo de vida útil de productos perecederos, conservando su calidad integral, la mejora de la presentación con envases y embalajes, la certificación y los análisis de higiene y sanidad, así como la reducción de pérdidas de postproducción, forman parte de los requerimientos de los productores.

Se ha identificado una relación de problemas y requerimientos de postcosecha, comunes en muchos casos, siendo: 1) Transferencia de tecnología. 2) Análisis y certificación. 3) Evaluación de nuevas variedades basadas en la biodiversidad. 4) Aumento del valor agregado de productos y subproductos. 5) Sanidad. 6) Calidad de los productos. 7) Normas técnicas. 8) Envases y embalajes. 9) Información técnica - económica. 10) Condiciones de transporte.

En nuestro país, en general es una realidad la falta de tecnologías de post-cosecha y tecnologías, que son necesarias para lograr una comercialización eficiente. Se sugiere entonces, la creación del Instituto de la Competitividad Provincial por ser de importancia estratégica, si se considera la necesidad de contar con tecnologías adecuadas que permitan solucionar los problemas específicos en las áreas de post-cosecha, transformación y comercialización, reforzando la actividad económica del sector agrario y agroindustrial, con productos homogéneos de alta calidad, de mayor valor agregado, en volúmenes suficientes, y la diversificación de los mercados.

g) Transformación de productos y subproductos. Dentro de este punto tenemos que el objetivo general consiste en disponer y transferir tecnologías de manejo y conservación adecuadas, que permitan el aprovechamiento de productos agropecuarios, agroexportables, así como de los recursos naturales, logrando el desarrollo de una eficiente comercialización, a través del fomento de la investigación y la mejora tecnológica.

Se busca pues, la modernización y la diversificación de las empresas, mediante la capacitación de sus asociados y la realización de proyectos de desarrollo tecnológico. Así, los objetivos específicos del proyecto, son: 1) El aumento de la vida útil de productos perecederos 2) El desarrollo de procesos o sistemas de procesamiento específicos 3) La disminución de los costos de producción y la mejora de la calidad 4) El incremento del ingreso de los agentes involucrados 5) El aumento de la oferta y la mejora del nivel nutricional. 
h) Marketing y desarrollo de nuevos productos. La comercialización de productos agropecuarios constituye, sin duda, un aspecto clave dentro de un proyecto integral para el desarrollo del agro y la agroindustria nacional.

El sector agrario y agroindustrial tienen, en la etapa de comercialización, la posibilidad de definir con éxito o fracaso toda la cadena productiva.

Con el apoyo a la exportación tradicional y no tradicional, y a la agroindustria, se espera poder obtener los siguientes "productos": 1) Incremento del volumen y el valor de nuestras exportaciones. 2) Disminución significativa de las pérdidas de postproducción. 3) Mejorar la calidad y dar mayor valor agregado a los productos. 4) Mejorar nuestra competitividad a nivel internacional. 5) Contribuir a mejorar la balanza comercial agropecuaria.

Muchos de los productos que pueden ser ofertados en los mercados de exportación tienen doble aplicación, es el caso de una serie de frutas nativas y plantas medicinales.

En ambos casos, pueden ser utilizadas en tanto alimentos y con fines curativos o preventivos de enfermedades, aparte del uso como saborizantes, perfumes y cosméticos.

Los productos denominados "nutracéuticos", funcionales o aquellos que son registrados en la categoría "FOSHU” (en Japón), tienen como principal característica ese doble propósito mencionado. En el caso de los llamados productos FOSHU (Foods for specified health use), cuya legislación es una de las más avanzadas, estos deben cumplir con una serie de requisitos antes de ser beneficiados para entrar en la categoría.

Los nueve criterios o requisitos que debe responder un alimento para acceder a la denominación FOSHU, son los siguientes: 1) El producto debe suponer contribuir a la mejora del régimen alimentario y al mantenimiento o mejora de la salud. 2) Las ventajas para la salud del producto o sus constituyentes se fundamentan sobre bases nutritivas médicas precisas.

3) Basados en conocimientos médicos y nutricionales, los niveles óptimos de consumo cotidiano deben poder ser definidos para el producto o sus constituyentes. 4) A partir de la experiencia adquirida, el consumo del producto o de sus constituyentes debe ser sin peligro. 5) Los constituyentes del alimento deben ser bien definidos en términos de propiedades físico - químicas y de análisis cualitativo y cuantitativo. 6) El producto no debe presentar déficits significativos en términos de constituyentes nutritivos en comparación a los productos alimenticios similares que no tiene uso en salud. 7) El producto debe ser similar a los alimentos consumidos habitualmente, más que a los consumidos ocasionalmente. 8) El producto debe presentarse bajo la forma de un alimento usual, y no bajo la forma de píldoras o tabletas. 9) El producto y sus constituyentes no tienen una finalidad exclusivamente médica.

i) Información. La información puede verse en dos niveles, con necesidades y estrategias particulares, en un primer caso general es necesario la preparación del inventario nacional y base de datos y desarrollo de la capacidad nacional en cultivos nativos promisorios, plantas medicinales, tintes y colorantes naturales, de pesticidas incluyendo el inventario y evaluación del potencial nacional con base en plantas y animales. Con esta acción, se busca efectivizar la investigación aplicada, la caracterización, la obtención y producción de material genético, tomar las medidas oportunas de conservación y prevención ambientales y una mayor eficiencia en el desarrollo de productos que agilicen la actividad productiva agroindustrial, actividad, que va desde el cultivo y crianza hasta la comercialización, y el consumidor.

De otro lado, la promoción de los productos es una herramienta que utiliza la difusión de información orientada al consumidor final y a los clientes intermediarios. La información escrita y audiovisual es de suma importancia para lograr posicionar los productos en los mercados objetivos. Muchas veces, debe recurrirse a la presentación directa de los productos a los consumidores (caso de las frutas tropicales exóticas en los supermercados), como estrategia, estos deberán poder degustarse y al mismo tiempo se dispondrá de boletines, o fascículos con información sobre las características, propiedades y formas de uso.

j) Ecología. El manejo de efluentes y desechos a lo largo de la cadena productiva y agroindustrial sería un primer nivel del accionar, en cuanto al plano ecológico se refiere.

Luego, se tiene que considerar la conservación y el sostenimiento de las especies, la variabilidad genética y el hábitat medio ambiental, cuya importancia es fundamental dentro del manejo de la ecología en su conjunto.

\section{Conclusiones}

El potencial agroindustrial de un país, una región o provincia, no sólo se basa en las ventajas comparativas de la biodiversidad y no se traducen necesariamente en ventajas competitivas, entre otras razones; también, por la falta de investigación y la explotación extractivas de materias primas con bajo valor agregado. Para revertir esta situación, es necesario el planteamiento de proyectos a largo plazo que contemplen el trabajo de investigación aplicada dirigido a superar cada uno de los puntos críticos identificados, a lo largo de la cadena agroindustrial. 
El inventario y evaluación del potencial agroindustrial de la provincia de Junín, permitirá tomar decisiones acertadas y oportunas, en cuanto a las diferentes políticas y estrategias de desarrollo agroindustrial (producción, evaluación e identificación, conservación, transformación, comercialización, etc.), en base a la información recopilada de las diferentes especies que conforman la biodiversidad de esta provincia.

El desarrollo de la provincia de Junín, tiene como motor principal el trabajo conjunto entre la biodiversidad y el potencial agroindustrial que posee esta parte de la región, siendo posible concluir que:

- Se ha adaptado ganado vacuno de doble propósito de la raza Brown Swiss.

- Posee ganado ovino de raza corriedale y raza Junín, apta para su aprovechamiento.

- Es necesario mejorar en manejo de los terrenos de conducción para animales.

- Es posible mejorar la producción agroindustrial de lácteos, carne, fibras, peletería y pequeña industria de cueros.

- Se han logrado identificar especies vegetales de gran potencial en la provincia.

De otra parte la sinergia originada por la Biodiversidad y la Agroindustria resulta fundamental, al sustentarse en ventajas reales que son alcanzadas a partir de las ventajas potenciales. Nos estamos refiriendo a beneficios concretos como:

- Menores costos por concepto de transporte, y menores volúmenes de contaminación.

- Mayor valor agregado y nivel de ingreso con el aprovechamiento comercial de nuestros productos autóctonos.

- Fomento de áreas de cultivos rentables, con manejo ecológico y la contribución a unaauténtica descentralización.

Como principal conclusión del presente informe, se puede afirmar que:

- La gran biodiversidad que presenta la provincia de Junín, hace posible mediante la inversión en transformación agroindustrial, al desarrollar toda su potencialidad dentro del marco de los 8 "Bionegocios", la obtención de cadenas productivas rentables, sostenibles, con una tasa ambiental de retorno positiva y la generación del empleo con desarrollo descentralizado en el mediano y largo plazo.

\section{Referencias bibliográficas}

Aksoy, M.A. (2005). The evolution of agricultural trade flows. En M. A Aksoy y J. Beghin, eds. Global agricultural trade and developing countries. Washington DC, Banco Mundial.

Banco Mundial. (2007). Informe para el desarrollo mundial 2008: Agricultura para el desarrollo. Washington, DC.

Boucher, F. y Riveros, H. (2000) Agroindustria y Agroindustria rural: Elementos conceptuales y de reflexión. IICA-Prodar. Perú.

CEPAL/FAOGTZ. (1998) Agroindustria y pequeña agricultura: Vínculos, potencialidades y oportunidades comerciales. Chile.

Da Silva, Carlos; Doyle, B.; Andrew; Shepherd, C.; Jenane y Miranda Da Cruz, Sergio. (2013) Agroindustrias para el desarrollo. FAO.

FAO (2007). Desafíos relativos al fomento de los agronegocios y la agroindustria. Comité de Agricultura, $20^{\circ} \mathrm{pe}-$ riodo de sesiones, 25- 28 de abril de 2007. Roma.

Ortega R. et al. (1995) Informe técnico. Subproyecto R2031. Conservación regional de raíces y rizomas en el sur del Perú. Periodo Abril 1994 - Julio 1995. Programa Colaborativo Biodiversidad de RTA’s Cuzco - Perú.

Programa Colaborativo Biodiversidad de Raíces y Tubérculos. (1995). Memorias 1993 - 1994. Consorcio para el Desarrollo Sostenible de la Ecorregión Andina. CONDESAN. La Molina.

Salazar Vera, Víctor. (1995) Documental Junín, el libro de la gratitud. Lima - Perú: Empresa Gráfica Baldeón.

Salazar Vera, Víctor. (1974) La altiplanicie de Junín en la historia. Lima - Perú: Imprenta Gamero. 11.

Tapia, M. (1990) Cultivos Andinos Sub - explotados y su aporte a la alimentación. FAO - INIAA.

Zapata Acha, Sergio. (2001) Posibilidades y potencialidad de la agroindustria en el Perú en base a la biodiversidad y los bionegocios. Documento de Trabajo.

RABOBANK. (2008). The boom beyond commodities: a new era shaping global food and agribusiness. Hong Kong, China. 